\title{
Effects of Fresh Yellow Onion Consumption on CEA, CA125 and Hepatic Enzymes in Breast Cancer Patients: A Double- Blind Randomized Controlled Clinical Trial
}

\author{
Farnaz Jafarpour-Sadegh ${ }^{1}$, Vahid Montazeri $^{2}$, Ali Adili ${ }^{3 \&}$, Ali Esfehani ${ }^{4 \&}$, \\ Mohammad-Reza Rashidi ${ }^{5}$, Mehran Mesgari ${ }^{6}$, Saeed Pirouzpanah ${ }^{1 *}$
}

\begin{abstract}
Onion (Allium cepa) consumption has been remarked in folk medicine which has not been noted to be administered so far as an adjunct to conventional doxorubicin-based chemotherapy in breast cancer patients. To our knowledge, this is the first study aimed to investigate the effects of consuming fresh yellow onions on hepatic enzymes and cancer specific antigens compared with a low-onion containing diet among breast cancer (BC) participants treated with doxorubicin. This parallel design randomized controlled clinical trial was conducted on $56 \mathrm{BC}$ patients whose malignancy was confirmed with histopathological examination. Subjects were assigned in a stratified-random allocation into either group received body mass index dependent 100-160 g/d of onion as high onion group (HO; $n=28$ ) or 30-40 g/d small onion in low onion group (LO; $n=28$ ) for eight weeks intervention. Participants, care givers and laboratory assessor were blinded to the assignments (IRCT registry no: IRCT2012103111335N1). The compliance of participants in the analysis was appropriate $(87.9 \%)$. Comparing changes throughout pre- and post-dose treatments indicated significant controls on carcinoembryonic antigen, cancer antigen-125 and alkaline phosphatase levels in the HO group $(\mathrm{P}<0.05)$. Our findings for the first time showed that regular onion administration could be effective for hepatic enzyme conveying adjuvant chemotherapy relevant toxicity and reducing the tumor markers in $\mathrm{BC}$ during doxorubicin-based chemotherapy.
\end{abstract}

Keywords: Breast cancer - onion - CEA - CA125 - hepatic enzyme - doxorubicin - intervention

Asian Pac J Cancer Prev, 16 (17), 7517-7522

\section{Introduction}

Doxorubicin (Adriamycin, DOX) is a potent antineoplastic anthracycline drug used in adjuvant therapy of wide a variety of malignancies (Arunachalam et al., 2013). However, there is a remarkable dose-dependent cardiotoxicity in concerning to DOX use, which can result life-threatening clinical relevance of heart failure (Outomuro et al., 2007). In addition to DOX-induced cardiotoxicity, the hepatotoxicity could also be attributed to DOX treatment (Injac et al., 2008; Bulucu et al., 2009). The mediated mechanism to explain DOX-induced hepatotoxicity is the generation of free radicals (Bulucu et al., 2009). Raising prevalence rate of DOX-treated malignancies given an essential intriguingly area to pave a right way to be capable to relief the undesired deleterious effects of DOX administration with less attenuating effects on the efficacy of drug.

One of the vulnerable cells to be influenced by DOX-induced oxidative stress are hepatocytes (Crib et al., 2005), suggesting that monitoring the functionality of liver may be specially involved in the evaluation of DOX-related damages generation in part (Dudka et al., 2012). Hence, there is a notable biochemical parameter like alkaline phosphatase (ALP) which is conventionally addressed to monitor possibly the pathologic features occurred in liver (Singh et al., 2013). The rising levels of serum ALP activities could also associate with poorer prognosis of metastasis in breast cancer (Singh et al., 2013). Evaluations of ALP with other biochemical parameters [such as aspartate aminotransferase (AST) and alanine aminotransferase (ALT)] are indicators of hepatocellula level of damages. They are considered as highly sensitive and fairly specific preclinical and clinical biomarker of hepatotoxicity (Ozera et al., 2008). They may thus be appropriate determinant variables in monitoring the progression of the disease and treatment efficiency (Tiwari et al., 2011; Singh et al., 2013).

There is unresolved research issue that how possibly could predict the risk of relapse and thus may benefit most

${ }^{1}$ Drug Applied Research Center, Also Department of Biochemistry and Dietetics, ${ }^{2}$ Department of Thoracic Surgery, Faculty of Medicine, Surgery Ward, Nour-Nejat Hospital, ${ }^{3}$ Clinic of Radiation Oncology, ${ }^{4}$ Hematology and Oncology Research Center, ${ }^{5}$ Faculty of Pharmacy, ${ }^{6}$ Drug Applied Research Center, Department of Biochemistry and Dietetics, Tabriz University of Medical Sciences, Tabriz,Iran \&Equal contributors*For correspondence: pirouzpanah@gmail.com,pirouzpanahs@tbzmed.ac.ir 
from adjuvant chemotherapy for breast cancer (McGuire and Clark, 1992; Mansour et al., 1994). Circulating tumor markers could be relatively helpful and carcinoembryonic antigen (CEA) and cancer antigen-125 (CA-125) have become widely used parameters in clinical follow-up care and monitoring therapy specified for breast cancer patients (Ebeling et al., 2002). Actually, they are non-invasive, reproducible for monitoring and easily bioavailable parameters which made them as strong independent prognostic determinants for overall- and disease-free survival in breast cancer patients (Ebeling et al., 2002; Pierga et al., 2012; Zhang et al., 2013).

Several cohort base follow-up studies have revealed that there are strong preventive effects of vegetables on breast cancer risk (Wang et al., 2009; Bradbury et al., 2014; Norat et al., 2014). A growing body of evidence on health promoting effects of Allium vegetables consumption have supported the preventive effects of these vegetables on neoplasm development, such as breast cancer (Challier et al., 1998; Norat et al., 2014; Tajaddini et al., 2015). Of Allium vegetables, onion is being used in traditional medicine and commonly used in Iranian daily dietary habit, has being paid more attention in epidemiologic studies from the standpoints of immunomodulatory, antiproliferative, antioxidant, anti-hormonal effects reported in experimental studies (Lanzotti, 2006; Benitez et al., 2011; Khaki et al., 2012; Elberry et al., 2014). Evidence from case-control studies have shown that a high frequency of onion consumption was associated with a notable decrease in BC risk (Leviac et al., 1993; Tajaddini et al., 2015). Although there is consensus throughout most observational studies to explicate an inverse association between onion intake and cancer risk, no clinical trial has to date supported significantly the controlling effect of onion consumption on growth-related biomarkers in connection with breast cancer prognosis.

Although the experimental studies have shown antioxidative and anticancer functions for active constituents in onion such as quercetin and diallyl trisulfide (Hosseinimehr et al., 2007; Pirouzpanah et al., 2009), to our knowledge, there is no interventional study was carried out to examine the effects of manipulating onion consumption in dietary content of breast cancer patients who treated with doxorubicin. Thereby, the aim of this double blinded randomized controlled clinical trial was to study the effects of raw yellow onion consumption on serum CEA, CA125 and hepatic enzymes in breast cancer who undergone doxorubicin-based chemotherapy.

\section{Materials and Methods}

\section{Study subjects}

This randomized, double-blind, placebo-controlled clinical trial study was conducted at Tabriz University of Medical Sciences (Faculty of Nutrition, Tabriz, Iran). BC patients whose disease had been approved histopathologically after radical or partial mastectomy in Nour-Nejat hospital and who referred to Shahid Ghazi Cancer Research Centre and private cancer clinics (Tabriz, Iran) aged 30 to 65 years old were enrolled of the primary population of women afflicted with BC (whole date range for patient recruitment was between October 2012 till June 2013). The inclusion criteria consisted of intently completing consent form prior to study, invasive ductal carcinoma (IDC), not being at stage IV (without metastasis), no history of any other malignancy, acute and chronic disease (such as severe liver or kidney failure, hyperthyroidism, polycystic ovary syndrome and gastrointestinal inflammatory disorders), allergy to onion, not being at pregnancy or lactation conditions, no prior history of chemo-, radio-, and hormone-therapy, no medical use of methotrexate and aspirin (Pirouzpanah et al., 2010). Subjects who received vitamin E and flaxseed supplement were excluded from the study. The eligible participants were requested to remain at their common habitual diet and lifestyle in the range of adherence to guidelines. Finally, after obtaining informed consent, fifty-six women with newly diagnosed BC fulfilled the selection criteria and were randomly assigned into either intervention (HO) or placebo (LO) group via block-random allocation (Figure 1). In this probability sampling method, the ratio of 2:1 in stratifying sampling frames was considered to define non-taxol chemotherapy protocols: (vs.) other chemotherapy regimens. The estimated sample size of fifty-six was preceded to mean difference (Adebamowo et al., 2005 ), and also confirmed by performing number needed to treat in diabetes (Adebamowo et al., 2005; Ebrahimi-Mamaghani et al., 2014). All procedures were subject to the prior approval of the Ethics Committee Center at Tabriz University of Medical Sciences (Ethics no: 5-4-6829). This clinical trial also received license permission from Iranian Registry of Clinical Trials linked to WHO Registry Network (IRCT no: IRCT no: IRCT2012103111335N1). The authors confirm that all the protocol of trials for this intervention was registered in the framework specified in IRCT homepage (http://www.irct.ir).

\section{Study design}

The second course of chemotherapy was considered the baseline of interventions and blood sampling was conducted prior to receive a second round of chemotherapy. Prior to the baseline of the study, all participants were placed at a two week run-in period (including the ovulation phase of menses cycle made change in run-in period time without breaking the blinding) in order to collect the information needed to be aware of chemotherapy toleration, lifestyle related risk factors and the respondent rate of patients to follow the basics of treatment. During the run-in period, participants were asked not to have additional onion and less than half a serving of Alliums vegetables (such as spring onion, shallots, garlic, garlic chives, native water-cress leaves and leek) on a daily basis. The amount of Allium had to be less than $90 \mathrm{~g} / \mathrm{d}$. An expert dietician met each participant individually during this time to undertake a primary dietary assessment (a three-day collection of 24-hour dietary record; for two weekdays and one for a typical weekend), teaching servings, limitations of dietary Allium vegetables, signs of intolerance to Alliums and simplifying the concept of treatment for reaching better adherence in eight weeks. The dietary records were created 
based on portion size guide estimated values in household utensils. All participants were individually counselled not to change their habitual diet, with some considerations regarding World Cancer Research Fund International (WCRF) guidelines (specifically, the fat content of their diet) (Wiseman, 2008; Pirouzpanah and Kouhdani, 2011; Ebrahimi-Mamaghani et al., 2014). Participants were also requested not to take any supplements (flax seed extract, soy products, fish liver oil, vitamin E, cartonoids and antioxidants) during the run-in period and also during the entire intervention. A physical activity record was also obtained from each participant during the run-in period, in addition to baseline assessments. Then, BC subjects included in the study were randomly allocated to either the intervention or control group by means of the method of sequence generation of computer-generated randomization software.

Overall, 46 patients who completed the trial received one of the following chemotherapy regimens: four cycles of intravenous (I.V.) doxorubicin every 3 weeks followed by three cycles of cyclophosphamide, methotrexate and 5-fluorouracil (CMF); four cycles of I.V. doxorubicin along with I.V. cyclophosphamide every 3 weeks, followed by three cycles of CMF; three cycles of I.V. doxorubicin every 3 weeks, followed by four cycles of I.V. docetaxel every 3 weeks, tracked by three cycles of CMF. The eight weeks intervention undertook till docetaxel was prescribed. Radiation therapy was also set as neoadjuvant therapy according to institutional guidelines.

Being respondent to onion intervention was evaluated by weighting the refrigerator-stored left onion at each three weeks visit appointed by who handed in onion packs. Compliance was also monitored by means of collected data from a weakly checklist in order to provide self-reporting of onion consumption in two daily meals. Each participant had three visits between the baseline and end point of eight weeks. In addition, physical examinations at these visits were conducted following chemotherapy-related changes such as cachexia. For not being respondent defined as who consumed was less than $85 \%$ (pure weight). At the beginning and end of the study, fasting blood samples were obtained and sera were stored at $-70^{\circ} \mathrm{C}$ until performing the analyses.

\section{Interventions}

At the baseline of the study, the BMI-dependent weighted onion (BMI $<24.9$ consumed $100-120 \mathrm{~g} / \mathrm{d}$ and BMI $>25$ consumed $140-160 \mathrm{~g} / \mathrm{d}$ ) was handed in for daily usage in high onion group (HO) beside the main meals (lunch and dinner). Participants in the low onion group (LO; placebo group) took 30 to $40 \mathrm{~g} / \mathrm{d}$ onion in addition to meals in a BMI-dependent manner. This superiority trial undertook to compare the treatment effects in HO group rather than LO group. All participants were requested not to consume any onion a day after receiving chemotherapy in order to lessen the possibility of fails in the respondent to the intervention. The duration of this intervention study for both groups was eight weeks. Two onions for daily usage was packed in 5 " $\times 5$ " white foam container (opaque plastic) to provide supplies every three weeks in order to fulfil the concealment criteria. Weight of container was adjusted by pieces of wood to make equal weigh sense and fixed by surrounding cotton (introduced to the protocol). Participants were asked to store all onions in a refrigerator at $4^{\circ} \mathrm{C}$. For consumption, only two outer layers had to be being peeled. Raw yellow onions were obtained from a local market (one seller, Tabriz), who declared obtaining the onions from a particular cultivated farm. Sequence generation and allocation concealment were listed and marked by designer of study and implemented by clinic personnel unaware of the allocated intervention at the time of enrolment. Participants, clinic personnel and laboratory assessors were blinded to the treatment assignments. In order to improve and partially achieve blinding, participants in different date of chemotherapy was included. Nutritionist IV software (version 3.5.2; 1994, N-Squared Computing, San Bruno, CA) was conducted to analyze the average of nutrients intake level for each participant from data obtained from24hour dietary records at baseline (Table 1). In addition, a validated food frequency questionnaire with 136 food items (Pirouzpanah et al., 2012; 2014a; 2014b) was used to evaluate the concordance of dietary data.

\section{Biochemical assessments}

Venous blood samples $(8 \mathrm{ml})$ were taken from subjects after at least 12 hours fasting and prior to a second round of chemotherapy in a clot tube (Vacuum Blood Collection Tube - Gel \& Clot Activator Tube, AMIS Medical Co., China) at Danesh Laboratory, where is under quality control and verified by the National Reference Laboratory (Tabriz, Iran). The samples were immediately centrifuged (Refrigerated Centrifuge, Sigma, Germany) at $3000 \times \mathrm{g}$ and at $20^{\circ} \mathrm{C}$ for $10 \mathrm{~min}$ to separate serum supernatant. Aliquots were stored at $-70^{\circ} \mathrm{C}$ until laboratory tests were conducted. Commercially available ELISA kits and standards were used as a follow-up to measure serum tumor markers, i.e., carcinoembryonic antigen (CEA) and cancer antigen 125 (CA 125) using a Diametra ELISA kit (CEA: DKO 051, and CA125: DKO 051; Via Pozzuolo, Italy). Serum aspartate aminotransferase (AST), alanine aminotransferase (ALT) and alkaline phosphatase (ALP) were determined using Pars-Azmoon kits (Tehran, Iran) at baseline and at the end of intervention to assess possible toxicities. The within and between assays' CVs for all biochemical measures were $<10 \%$. For each biomarker, measures were performed at the same time in one laboratory run. The name of the patient in each sera sample was labelled with a specific numeric code. ALT below the 1.5 times of normal upper limit $(<35 \mathrm{U} / \mathrm{L} ; \mathrm{n}=30)$ considers as chemotherapeutic toxicity (introduced to the protocol; $\mathrm{n}=0$ ).

\section{Statistical analyses}

A Kolmogorov-Smirnov test was performed to ensure the normality of data distribution in each analysed subclass. A graphed linear histogram was used to examine the skewness and kurtosis. A box plot was used to detect outliers. Descriptive results were expressed in mean \pm standard deviation (S.D.) and median (95\%CI) for the general characteristics of studied variables. Data that were not normally distributed were analysed using 
nonparametric tests. Two independent sample t-tests were used to compare variables between the placebo and intervention groups. With-in group comparisons in an interventional arm between the baseline and eight weeks' intervention were carried out by paired t-test. For each comparison, $\mathrm{P}<0.05$ was considered as statistically significant. All statistical analyses were performed using SPSS software (ver.15).

\section{Results}

\section{Demographic characteristics}

Table 1. Demographic and Clinical Characteristics of BC Patients in LO (placebo; $n=23$ ) and HO (intervention; $\mathbf{n = 2 3}$ ) Groups at Baseline of Intervention

\begin{tabular}{|c|c|c|c|}
\hline Characteristics & $\begin{array}{c}\text { LO } \\
\text { (Placebo, } \\
n=23 \text { ) } \\
\text { Mean } \pm \text { S.D. }\end{array}$ & $\begin{array}{c}\mathrm{HO} \\
\text { (Intervention } \\
\mathrm{n}=23 \text { ) } \\
\text { Mean } \pm \text { S.D. }\end{array}$ & P-value* \\
\hline \multicolumn{4}{|l|}{ Age (years) } \\
\hline At diagnosis & $42.7 \pm 5.9$ & $43.9 \pm 8.7$ & 0.57 \\
\hline At first delivery & $21.7 \pm 3.6$ & $22.1 \pm 3.7$ & 0.937 \\
\hline At first menses & $13.9 \pm 1.3$ & $13.5 \pm 1.7$ & 0.455 \\
\hline $\mathrm{BMI}\left(\mathrm{kg} / \mathrm{m}^{2}\right)$ & $27.4 \pm 4.8$ & $27.8 \pm 3.7$ & 0.832 \\
\hline \multicolumn{4}{|l|}{ Daily dietary intake } \\
\hline Total calorie intake (kcal/day & y) $1893 \pm 417$ & $1952 \pm 442$ & 0.641 \\
\hline Protein intake (g/day) & $68.6 \pm 21.6$ & $67.9 \pm 17.5$ & 0.918 \\
\hline Carbohydrate intake (g/day) & $253 \pm 81$ & $270 \pm 98$ & 0.505 \\
\hline Fat intake (g/day) & $56.5 \pm 20.75$ & $56.6 \pm 18.8$ & 0.977 \\
\hline Total dietary fiber (g/day) & $4.6 \pm 2.9$ & $4.6 \pm 2.9$ & 0.956 \\
\hline Soluble fiber (g/day) & $0.6 \pm 0.5$ & $0.5 \pm 0.7$ & 0.286 \\
\hline Crude fiber (g/day) & $3.9 \pm 2.5$ & $4.1 \pm 2.4$ & 0.684 \\
\hline
\end{tabular}

BMI, body mass index; * Independent sample t-test was performed
At last 23 patients in each group completed the study. Mean age at diagnosis for participants included in the study was $42.7 \pm 5.9$ years (range: 32.0 to 58.0 years) for the LO group and $43.9 \pm 8.7$ years (range: 30.0 to 63.0 years) for the intervention group (HO). The general and dietary characteristics of the BC participants at the baseline of the study are summarized in Table 1 . Two groups were similar with respect to demographic variables and wellknown risk factors for BC (Table 1). Daily intakes of total energy, carbohydrates, fat, protein and fibre contents did not differ significantly between the two groups at baseline (Table 1). The habitual dietary and lifestyle-related factors of all the subjects did not differ significantly in terms of the interventions. The compliance of participants in the analysis was as high as $87.85 \%$.

\section{Intervention effect}

Raw yellow onion was administered to adjuvanttreated BC patients consistently and begun in the post-second course of chemotherapy (after two days) in a double-blind state. Table 2 summarised average serum levels of variables in terms of cancer specific antigens and hepatic enzymes at baseline compartment of study and 8 weeks after the intervention in women with $\mathrm{BC}$ who received onion (HO group) versus LO consumers. Mean serum AST, ALT and ALP levels did not change statistically significant between the placebo and intervention groups after eight weeks. Two indicators of liver toxicity, ALT and AST, increased approximately in each group; however, the elevation of AST in the placebo group was higher than in the intervention $(26.08 \pm 9.82$ to $34.26 \pm 14.58 \mathrm{IU} / \mathrm{L} ; \mathrm{P}=0.05)$, in spite of a statistically

Table 2. Serum Levels of Cancer Specific Antigens and Hepatic Enzymes at Baseline Compartment of Study and 8 weeks After the Intervention in Women with BC Who Received Onion (HO group) versus LO Consumers

\begin{tabular}{|c|c|c|c|c|c|}
\hline Variable & & $\begin{array}{c}\text { LO } \\
(\text { Placebo, } \mathrm{n}=23) \\
\text { Mean } \pm \text { S.D. }\end{array}$ & $\begin{array}{c}\mathrm{HO} \\
\text { (Intervention, } \mathrm{n}=23 \text { ) } \\
\text { Mean } \pm \text { S.D. }\end{array}$ & Mean difference & P-value ${ }^{a}$ \\
\hline \multirow[t]{4}{*}{$\mathrm{CEA}^{\mathrm{c}}$} & Pre-dose & $2.73 \pm 1.33$ & $3.10 \pm 2.58$ & -0.37 & 0.817 \\
\hline & Week 8 & $2.61 \pm 1.58$ & $2.49 \pm 1.85$ & -0.12 & 0.823 \\
\hline & Mean difference & 0.18 & 0.69 & & \\
\hline & $\mathrm{P}$-value ${ }^{\mathrm{b}}$ & 0.548 & 0.023 & & \\
\hline \multirow[t]{4}{*}{$\log \mathrm{CA} 125^{\mathrm{d}}$} & Pre-dose & $18.53 \pm 8.94$ & $21.13 \pm 16.64$ & -2.59 & 0.513 \\
\hline & Week 8 & $14.64 \pm 7.95$ & $16.58 \pm 16.21$ & -1.94 & 0.616 \\
\hline & Mean difference & 4.33 & 4.93 & & \\
\hline & $\mathrm{P}$-value & 0.067 & 0.04 & & \\
\hline \multirow[t]{4}{*}{$\log \mathrm{ALT}^{\mathrm{e}}$} & Pre-dose & $25.65 \pm 23.37$ & $25.56 \pm 16.38$ & 5.95 & 0.988 \\
\hline & Week 8 & $37.26 \pm 20.66$ & $26.87 \pm 18.25$ & 5.74 & 0.078 \\
\hline & Mean difference & 11.61 & 1.3 & & \\
\hline & P-value & 0.086 & 0.611 & & \\
\hline \multirow[t]{4}{*}{$\log \mathrm{AST}^{\mathrm{f}}$} & Pre-dose & $26.08 \pm 9.82$ & $31.43 \pm 12.31$ & -5.34 & 0.111 \\
\hline & Week 8 & $34.26 \pm 14.58$ & $30.91 \pm 16.29$ & 3.34 & 0.467 \\
\hline & Mean difference & 8.17 & 0.52 & & \\
\hline & P-value & 0.051 & 0.872 & & \\
\hline \multirow[t]{4}{*}{ ALP g } & Pre-dose & $155.81 \pm 33.92$ & $143.30 \pm 49.95$ & 12.51 & 0.333 \\
\hline & Week 8 & $150.74 \pm 35.53$ & $133.40 \pm 49.73$ & 17.33 & 0.184 \\
\hline & Mean difference & -3.91 & -9.68 & & \\
\hline & P-value & 0.57 & 0.41 & & \\
\hline
\end{tabular}

*LO, low onion group; HO, high onion group. Data are expressed in geometric mean \pm S.D., whereas CA125, ALT and AST are presented in arithmetic mean; ${ }^{a}$ Independent sample t-test was performed between group; ${ }^{\mathrm{b}}$ Paired t-test was performed to compare within changes in intervention group during the study; ${ }^{\mathrm{c}}$ Carcinoembryonic antigen; ${ }^{\mathrm{d}}$ Cancer antigen $125 ;{ }^{\mathrm{e}}$ Alanine aminotransferase

${ }^{\mathrm{f}}$ Aspartate aminotransferase; ${ }^{\mathrm{g}}$ Alkaline phosphatase (ALP) 


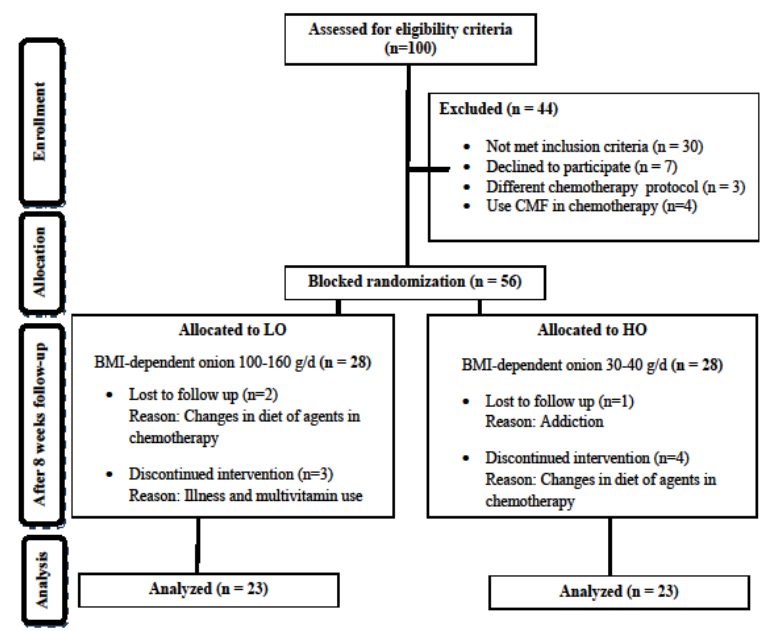

Figure 1. CONSORT Flow Chart Diagram of Intervention

non-significant result.

During the study, serum ALP as a biomarker of cardiotoxicity showed a non-significant reduction that was higher in the HO group (143.09to $133.40 \mathrm{IU} / \mathrm{L}$ ). A CEA decrease during treatment was a remarkable change obtained only in the high onion feed group $(\mathrm{P}<0.05)$. Likewise, CA125 also showed a notable decline in the HO group $(\mathrm{P}<0.05)$.

\section{Discussion}

Findings from this randomized controlled clinical trial conducted on newly diagnosed $\mathrm{BC}$ patients receiving postoperative chemotherapy and raw yellow onion intervention concurrently showed favourable effects in terms of decreasing certain tumor markers.

Although sera levels of ALT, AST, ALP enzymes apparently increased in LO group during intervention; however, in the $\mathrm{HO}$ group they were practically unchanged and ALP levels decreased non-significantly. These findings are consistent with those studies previously conducted (Obioha et al., 2009; Dudka et al., 2012). Resveratrol administration (RV) concomitantly with doxorubicin (DOX; once a week over a period of seven weeks), showed no significant difference in serum activity of AST, ALT and ALP between the DOX and DOX+RV groups (Dudka et al., 2012). However, Obioha et al. (2009) indicated the hepatoprotective effect of onion extracts on cadmium (Cd)-induced oxidative damage in rats (Obioha et al., 2009). Cd is a toxic pollutant that has been addressed as possibly increasing the plasma levels of ALT and AST. Onion extracts have shown to significantly attenuate these adverse effects of $\mathrm{Cd}$, apparently in a dose-dependent manner and seem to exert potent hepatoprotective effects (Obioha et al., 2009). Although, the hepatoprotective effects of onion has been scarcely noticed thus far, the present study suggested the controlling effects of onion consumption on sera levels of hepatic enzymes, particularly on ALP apparently might mask the DOXrelated enzymatic changes of liver during intervention.

According to our findings, onion treatment significantly decreases the plasma levels of CEA and CA-125. Although to the best of our knowledge, no study has to date evaluated the hypothesis of tumour marker changes regarding high onion consumption, our findings put forth the possibility of the regulatory effects of onion consumption on the prognosis of $\mathrm{BC}$ alongside treatment with DOX over a shorter timeframe. Accordingly, there is a meta-analysis undergone on different population-based studies for gastric cancer risk assessment in Korea which also represented the protective association for Allium vegetables (Woo et al., 2014).

There were some possible limitations in present study. The sample size assigned to each tail of intervention group was relatively small. To provide homogenous adjuvant chemotherapy which was co-administered in this interventional study, there was an attempt to consider only DOX-base regimens. We suggest that at least stratified random allocation could be helpful to weaken the variation conveyed based on chemotherapy type. Although chemotherapy related metabolic changes influence weight loss is an impressive secondary event after receiving the 1st course of chemotherapy, underwent intervention after the 2nd course of regimen was not associated with significant reduced anthropometric changes in controls.

In conclusion, the present study demonstrated the effectiveness of onion to control CEA and CA125 tumor markers. But they were not significant between groups at the end of study among $\mathrm{BC}$ patients during doxorubicinbased chemotherapy. Manipulation of diet through high intake of onion is promising to possibly improve a synergistic effect on DOX-based chemotherapy.

\section{Acknowledgements}

The authors would like to thank all the subjects involved in this study for their participation and the staff members of Nour-Nejat Hospital, Cancer Clinic, Danesh-2 laboratory, Shahid Ghazi and Emam Reza (peace be upon him) Hospitals for spending their time to this research. This article was outlined using a dataset obtained from a M.Sc. thesis titled "The effect of raw yellow onion consumption on plasma levels of hormonal/ metabolic parameters in patients with primary breast cancer: A randomized controlled clinical trial", registered at Tabriz University of Medical Sciences (Registration no.: 5/97/803).The authors' responsibilities were as follows S.P., and V.M.: designed research; S.P., F.J.S., A.A., A.E. and M.R.R.: conducted research and experiments; S.P., M.R.R. and F.J.S.: analyzed data; and S.P., V.M., M.R.R. and F.J.S.: wrote manuscript.

\section{References}

Adebamowo CA, Cho E, Sampson L, et al (2005 ). Dietary flavonols and flavonol-rich foods intake and the risk of breast cancer. Int J Cancer, 114, 628-33.

Arunachalam S, Tirupathi Pichiah PB, Achiraman S (2013). Doxorubicin treatment inhibits pparc and may induce lipotoxicity by mimicking a type 2 diabetes-like condition in rodent models. FEBS Letters, 587, 105-10.

Benitez V, Molla E, Martín-Cabrejas MA, et al (2011). Characterization of industrial onion wastes (Allium cepa L.): dietary fibre and bioactive compounds. Plant Foods Hum Nutr, 66, 48-57 
Farnaz Jafarpour-Sadegh et al

Bradbury KE, Appleby PN, Key TJ (2014). Fruit, vegetable, and fiber intake in relation to cancer risk: findings from the European Prospective Investigation into Cancer and Nutrition (EPIC). Am J Clin Nutr, 100, 394S-8S.

Bulucu F, Ocal R, Karadurmus N, et al (2009). Effects of $\mathrm{N}$-acetylcysteine, deferoxamine and selenium on doxorubicin- induced hepatotoxicity. Biol Trace Elem Res. 132, 184-96.

Challier B, Perarnau JM, Viel JF (1998). Garlic, onion and cereal fibre as protective factors for breast cancer: a french casecontrol study. Eur J Epidemiol, 14, 737.

Crib AE, Peyrou M, Muruganandan S, et al (2005). The endoplasmic reticulum in xenobiotic toxicity. Drug Metab Rev, 37, 405-42.

Dudka J, Gieroba R, Korga A, et al (2012). Different effects of resveratrol on dose-related doxorubicin-induced heart and liver toxicity. Evid Based Complement Alternat Med, 1-10.

Ebeling FJ, Stieber P, Untch M, et al (2002). Serum CEA and CA15-3 as prognostic factors in primary breast cancer. Brit J Cancer, 86, 1217-22.

Ebrahimi-Mamaghani M, Saghafi-Asl M, Pirouzpanah S, et al (2014). Effects of raw red onion consumption on metabolic features in overweight or obese women with polycystic ovary syndrome: a randomized controlled clinical trial. J Obstet Gynaecol Res, 40, 1067-76.

Elberry AA, Mufti S, Al-Maghrabi J, et al (2014). Immunomodulatory effect of red onion (Allium cepa Linn) scale extract on experimentally induced atypical prostatic hyperplasia in Wistar rats. Mediators Inflamm, 2014, 640746.

Hosseinimehr SJ (2007). Trends in the development of radioprotective agents. Drug Discov, 12, 794-805.

Hosseinimehr SJ, Azadbakht M, Mousavi SM, et al (2007). Radioprotective effects of hawthorn fruit extract against c-irradiation in mouse bone marrow cells. $J$ Radiat Res, 48, 63-8.

Injac R, Boskovic M, Perse M, et al (2008). Acute doxorubicin nephrotoxicity in rats with malignant neoplasm can be successfully treated with fullerenol $\mathrm{C} 60(\mathrm{OH}) 24$ via suppression of oxidative stress. Pharmacol Rep, 60, 742-9.

Khaki A, Farnam A, Davatgar-Badie A, et al (2012). Treatment effects of onion (Allium cepa) and ginger (Zingiber officinale) on sexual behavior of rat after inducing an antiepileptic drug (lamotrigine). Balkan Med J, 29, 236-42.

Lanzotti V (2006). The analysis of onion and garlic. $J$ Chromatogr, 1112, 3-22.

Leviac F, La Vecchiacd C, Gulieb C, et al (1993). Dietary factors and breast cancer risk in Aaud, Switzerland. Nutr Cancer, 19, 327-35.

Mansour EG, Ravdin PM, Dressier L (1994). Prognostic factors in early breast carcinoma. Cancer Metab, 74, 381

McGuire WL, Clark GM (1992). Prognostic factors and treatment decisions in axillary-node-negative breast cancer. $N$ Engl J Med, 326, 1756-61.

Norat T, Aune D, Chan D, et al (2014). Fruits and vegetables: updating the epidemiologic evidence for the WCRF/AICR lifestyle recommendations for cancer prevention. Cancer Treat Res, 159, 35-50.

Obioha UE, Suru SM, Ola-Mudathir KF, et al (2009). Hepatoprotective potential of onion and garlic extracts on cadmium-induced oxidative damage in rats. Biol Trace Elem Res, 129, 143-56.

Outomuro D, Grana DR, Azzato F, et al (2007). Adriamycininduced myocardial toxicity: new solutions for an old problem? Int J Cardiol, 117, 6-15.

Ozera J, Ratner M, Shaw M, et al (2008). The current state of serum biomarkers of hepatotoxicity. Toxicology, 245, 194-205.
Pierga JY, Hajage D, Bachelot T, et al (2012). High independent prognostic and predictive value of circulating tumor cells compared with serum tumor markers in a large prospective trial in first-line chemotherapy for metastatic breast cancer patients. Ann Oncol, 23, 618-24.

Pirouzpanah S, Koohdani F (2011). Nutritional facts about macronutrients in cancer. In: Mehdipour P. (ed.) Brid Cell Biol Genetics Cancer Clin, 59-82.

Pirouzpanah S, Taleban FA, Atri M, et al (2010). The effect of modifiable potentials on hypermethylation status of retinoic acid receptor-beta2 and estrogen receptor-alpha genes in primary breast cancer. Cancer Causes Control, 21, 2101-11.

Pirouzpanah S, Taleban FA, Mehdipour P, et al (2014a). The biomarker-based validity of a food frequency questionnaire to assess the intake status of folate, pyridoxine and cobalamin among Iranian primary breast cancer patients. Eur J Clin Nutr, 68, 316-23.

Pirouzpanah S, Taleban FA, Sabour S, et al (2012). Validation of food frequency questionnaire to assess folate intake statues in breast cancer patients. Razi J Med Sci, 18, 31-41.

Pirouzpanah S, Taleban FA, Mehdipour P, et al (2014b). Plasma total homocysteine level in association with folate, pyridoxine, and cobalamin status among Iranian primary breast cancer patients. Nutr Cancer, 66, 1097-108.

Singh AK, Pandey A, Tewari M, et al (2013). Advanced stage of breast cancer hoist alkaline phosphatase activity: risk factor for females in India Biotech, 3, 517-20.

Tajaddini A, Pourzand A, Sanaat Z, Pirouzpanah S (2015). Dietary resistant starch contained foods and breast cancer risk: a case-control study in northwest of Iran. Asian Pac J Cancer Prev, 16, 4185-92.

Tiwari DK, Jin T, Behari J (2011). Bio-distribution and toxicity assessment of intravenously injected anti-HER2 antibody conjugated $\mathrm{CdSe} / \mathrm{ZnS}$ quantum dots in Wistar rats. Int $J$ Nanomedicine, 6, 463-75.

Wang L, Lee IM, Zhang SM, et al (2009 ). Dietary intake of selected flavonols, flavones, and flavonoid-rich foods and risk of cancer in middle-aged and older women. Am J Clin Nutr, 89, 905-12.

Wiseman M (2008). The second World Cancer Research Fund/ American Institute for Cancer Research expert report. Food, nutrition, physical activity, and the prevention of cancer: a global perspective. Proc Nutr Soc, 67, 253-6.

Woo HD, Park S, Oh K, et al (2014). Diet and cancer risk in the Korean population: a meta- analysis. Asian Pac J Cancer Prev, 15, 8509-19.

Zhang SJ, Hu Y, Qian HL, et al (2013). Expression and significance of ER, PR, VEGF, CA15-3, CA125 and CEA in judging the prognosis of breast cancer. Asian Pacific $J$ Cancer Prev, 14, 3937-40. 